\title{
A Selective Aperiodic Checkpointing Approach for VLR Failure Restoration in Wireless Mobile Networks
}

\author{
Md. Mohsin Ali, Mst. Shakila Khan Rumi, Md. Hasnat-E-Rabbi, and M. M. Zakariya \\ Department of Computer Science and Engineering (CSE) \\ Khulna University of Engineering \& Technology (KUET), Khulna - 9203, Bangladesh \\ mohsin_kuet_cse@yahoo.com, shakila_rumi@yahoo.com, shuvocse@gmail.com, \\ zakariya002@gmail.com
}

\begin{abstract}
This paper proposes a new selective aperiodic checkpointing approach for VLR (Visitor Location Register) failure restoration in wireless mobile networks. In this approach, the VLR data are backed up in the non-volatile storage whenever the location update counter for that VLR exceeds the pre-determined threshold. It restores the backed up VLR data after the failure of the VLR and sends location confirmation request messages only to the selected Registration Areas (RAs) whose information is updated between the time period of back up and failure of the VLR. This is necessary as this information is obsolete in the backed up VLR for this reason and needs to be updated in the restored VLR. However, previous work on aggressive restoration based on periodic and aperiodic checkpointing also provide solutions for VLR database failure recovery. But these solutions are not feasible as they broadcast the location confirmation request messages to the RAs whose location information is found to be obsolete during the restoration. The analytical model and experimental results also show that the proposed method outperforms other previous approaches as it selectively sends the location confirmation request messages instead of broadcasting to the RAs during VLR restoration process.
\end{abstract}

\section{Categories and Subject Descriptors}

D.3.3 [Programming Languages]: Language Contructs and

Features - abstract data types, polymorphism, control structures.

\section{General Terms}

Mobile Computing, Wireless Communications.

\section{Keywords}

Periodic checkpointing, aperiodic checkpointing, location update, VLR, RA, failure restoration.

\section{INTRODUCTION}

Location management in wireless mobile networks is defined as the tracking of MTs in the network coverage area. In order to track the location of these roaming MTs', two types of standards are recently used in all wireless mobile networks such as basic IS-41 [1] and GSM [2]. Both of these standards are based on a two-level hierarchy of location databases called Home Location Register (HLR) and VLR used to store and maintain the MTs' current location information. The whole network coverage area of these standards is separated into cells having same size and shape. Each cell has a Base Transceiver Station (BTS) in its center through which MTs of this cell communicate through a wireless link. The cells are grouped together to form larger areas called Registration Areas (RAs). All the BTSs within the RA are in wire-connected to a Mobile Switching Center (MSC) and the MTs are wireless- connected to the BTSs. These MSCs are wire-connected to one or more HLRs in the network depending on its configuration. An HLR is the centralized database storing all the MTs' location information for the whole network. Similarly, each VLR stores location information of the MTs currently residing in its corresponding RA.

Two basic operations in wireless mobile networks are location registration and call delivery. When a subscriber roams from one RA to another, a procedure is performed for informing the MT's new location information to update in the HLR. On the other hand, a call delivery requires the HLR to query the VLR to find the MT's current location information. If the VLR mobility database fails, it becomes quite impossible to deliver the call to the subscribers as well as to perform location registration. As a result, VLR mobility database recovery is very much necessary after the failure to assure the continuous service availability to subscribers without any disruption of communication. An extensive work has been done on mobility database failure recovery and studied in [1], [2], [3], [4], $[5],[6]$.

In this paper, we propose a selective aperiodic checkpointing approach for VLR database failure restoration. In this approach, the VLR databases are saved aperiodically when it exceeds the location update counter threshold. After the failure, the VLR restores its saved database and sends location confirmation request messages only to the selected RAs whose location information is obsolete due to location updates between the time of database saving and database failure.

The rest of the paper is organized as follows. Section 2 provides an overview of the related recent research work. Our proposed approach is described in section 3. Section 4 provides the analytical modeling and comparison among different approaches based on some experimental results. We provide a concluding remark in section 5 .

\section{RELATED WORK}

A lot of work has been done on VLR database failure restoration to make the service continuously available to its subscribers. A basic IS-41 scheme is proposed in [1] where the HLR and VLRs are not backed up in the non-volatile storages. After a failure, the VLRs reconstruct their MTs' location information databases during the registration message exchange between the MTs and the HLR.

A GSM scheme is proposed in [2] where both the HLR and VLRs are backed up periodically in the non-volatile storages. The VLRs restored their databases from their corresponding non-volatile storages after the failure. Although the HLR may aggressively restore its databases by sending location request messages to the known VLRs, but the VLRs cannot do this to the RAs to aggressively restore their databases. 


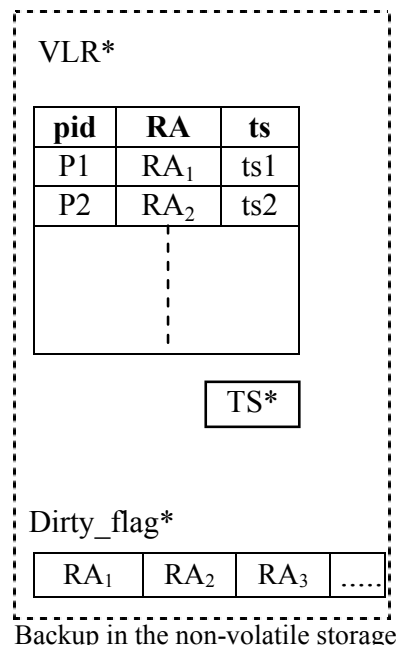

VLR

\begin{tabular}{|c|c|c|c|}
\hline pid & TMSI & $\mathbf{R A}$ & ts \\
\hline P1 & tmsil & $\mathrm{RA}_{1}$ & ts 1 \\
\hline P2 & tmsi2 & $\mathrm{RA}_{2}$ & ts2 \\
\hline & 0 & & \\
\hline & & & $\mathrm{TS}$ \\
\hline
\end{tabular}

Counter

\begin{tabular}{l|l|l|l|}
$\mathrm{RA}_{1}$ & $\mathrm{RA}_{2}$ & $\mathrm{RA}_{3}$ & $\ldots .$. \\
\hline
\end{tabular}

Figure 1. The data structures used to recover the VLR.

The HLR and VLR restoration with periodic HLR and VLR checkpointing together with periodic location update is proposed in [3]. It periodically backs up the VLR databases to the non-volatile storages. After the failure, it restores the VLR databases and recovers the obsolete information by periodic location update from the MTs. This periodic checkpointing and location update increases the paging although reduces the VLR restoration cost.

The periodic checkpointing of the HLR and VLR databases with location update on demand is proposed in [4]. In this approach, the VLR databases are periodically backed up to the non-volatile storages like [3]. But it recovers the obsolete information from the restored VLR databases by sending location update message on demand when the failure occurs. This approach is a little bit better than [3] as it sends location update messages from MTs to the VLR only during the occurrence of failure instead of sending periodically.

An aperiodic checkpointing of the HLR and VLRs with location update on demand is proposed in [5]. In this scheme, VLR databases are backed up in the non-volatile storages at the time of exceeding the location update counter of the corresponding VLR to a threshold value. These databases are restored immediately to the corresponding VLRs after the failure. Additionally, a location request message is broadcasted to the RAs to send the MTs' location information to the concerned VLR to update the obsolete information. This aperiodic approach reduces the unnecessary VLR checkpointing when the MTs not performing location update to the VLRs. But it broadcasts the location update message to all the RAs although the location information for all these RAs is not obsolete.

A broadcast approach for Universal Mobile Telecommunications System (UMTS) is proposed in [6] where a broadcast message is issued in the network to recover the mobility databases after the failure. Several parameters are considered to analyze the performance of this approach.

\section{PROPOSED APPROACH}

In our proposed selective aperiodic checkpointing approach, a data structure called Counter (an array of counters) is used to count the number of location update performed from each RA to the VLR. When the counter for any of the RAs exceeds its threshold, the VLR database is checkpointed or saved in the non-volatile storage. This database is restored in the VLR from this non-volatile storage when it fails. But some of the location information found there may be obsolete as MTs may perform location update between the time of checkpointing and its failure. The data structures called Dirty_flag* (an array of flags) and TS* (the last checkpointing time) are used to keep track of the RAs whether their backup location information is obsolete or not and the last VLR checkpointing time, respectively. The VLR sends location confirmation request message to the selected RAs whose backup information is obsolete (Dirty_flag* is set). The MTs send location confirmation message to the VLR through RA if it updates its location later than TS* after receiving the location request message from the RA. In this way, the VLR database is restored on it after the failure and the probability of collisions is reduced since the number of MTs and RAs involved in location confirmation is significantly smaller than the previous approaches. Figure 1 shows the data structures and Table 1 shows the terms and symbols used to describe the algorithm of the proposed method. The proposed aperiodic VLR restoration algorithm is shown in Figure 2.

Table 1. Terms or symbols used in proposed algorithms.

\begin{tabular}{|c|c|}
\hline $\begin{array}{l}\text { Term or } \\
\text { Symbol }\end{array}$ & Meaning \\
\hline VLR & $\begin{array}{l}\text { The VLR in which the MT is currently located } \\
\text { (the MT entered this VLR at time ts) }\end{array}$ \\
\hline pid & The permanent identity of the MT \\
\hline TMSI & $\begin{array}{l}\text { The current temporary Identification (ID) of } \\
\text { the MT assigned by the VLR }\end{array}$ \\
\hline RA & $\begin{array}{l}\text { The current registration area (RA) the MT is } \\
\text { visiting (the MT entered this RA at time ts) }\end{array}$ \\
\hline ts & The time stamp of the MT's latest registration \\
\hline TS & $\begin{array}{l}\text { The last database backup (checkpointing) time } \\
\text { of the VLR }\end{array}$ \\
\hline Counter & $\begin{array}{l}\text { An array of location update counters in the } \\
\text { RAs }\end{array}$ \\
\hline Dirty_flag* & $\begin{array}{l}\text { An array of flags that identify whether or not } \\
\text { the VLR backups for the RAs are obsolete or } \\
\text { not saved in the non-volatile sorage }\end{array}$ \\
\hline $\mathrm{N}_{\mathrm{v}}$ & $\begin{array}{l}\text { A threshold value for VLR checkpointing } \\
\text { process }\end{array}$ \\
\hline VLR* & $\begin{array}{l}\text { The backup of the VLR in the non-volatile } \\
\text { storage }\end{array}$ \\
\hline TS* & $\begin{array}{l}\text { The backup of the last database backup } \\
\text { (checkpointing) time of the VLR in the non- } \\
\text { volatile storage }\end{array}$ \\
\hline
\end{tabular}




\section{Procedure VLR REGISTRATION}

(Registration message handling)

IF $V L R=V L R_{\text {old }}$ THEN $/ *$ Intra-VLR $* /$

-Find the portable $\mathrm{p}$ that satisfies $V L R[p] . T M S I=t m s i$.

ELSE /*Inter-VLR*/

-Send a request for the portable's identity to the old $V L R$.

-Get the portable identity $p$.

-Create a new record for the portable.

-Inform the HLR of the registration.

(TMSI assignment)

-Generate a new TMSI, tmsi $i_{\text {new }}$, for the portable.

(Location update)

-Set $V L R[p]$. ts $=t$.

-Set $V L R[p] . T M S I=$ tmsi $_{\text {new }}$

-Set $V L R[p] . R A=R A_{\text {new }}$.

(Counter update)

- Set Counter $\left[R A_{\text {new }}\right]=\operatorname{Counter}\left[R A_{\text {new }}\right]+1$.

IF Counter $\left[R A_{\text {new }}\right]=1$ THEN

-Set dirty_flag* $\left[R A_{\text {new }}\right]=1$.

(Checkpointing)

IF Counter $\left[R A_{\text {new }}\right]>N_{v}$ THEN

-Trigger a VLR checkpointing process.

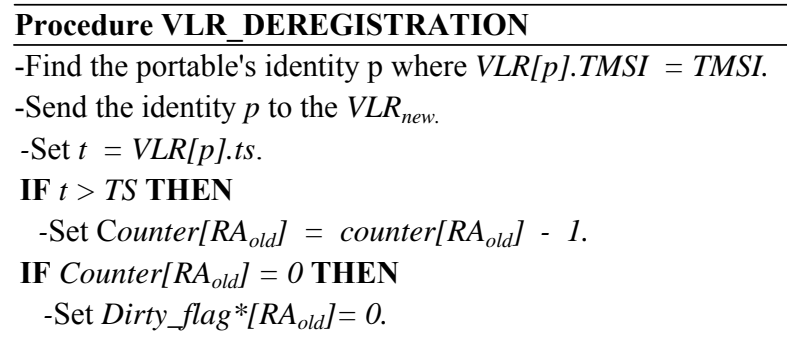

\section{Procedure VLR CHECKPOINTING}

-Set $V L R^{*} \leftarrow V L R$.

-Set $T S^{*} \leftarrow T S$.

FOR all $R A_{i}$ DO

-Set Counter $\left[R A_{i}\right]=0$.

FOR all $R A_{i}$ DO

-Set Dirty_flag* $\left[R A_{i}\right]=0$.

\section{Procedure VLR_RESTORATION}

(Restore the backup VLR which are checkpointed previously)

-Set $V L R \leftarrow V L R *$.

FOR all $R A_{i}$ DO

IF Dirty_flag* $\left[R A_{i}\right]=1$ THEN

(execute a confirmation algorithm)

-VLR sends location confirmation request message to the $R A_{i \text {. }}$

-MTs confirm their location information to the VLR through

$R A_{i}$ which perform handoff or location update after TS*.

Figure 2. Proposed aperiodic VLR restoration algorithm.
Table 2. Terms or symbols used in analytical modeling.

\begin{tabular}{|c|c|}
\hline $\begin{array}{l}\text { Term or } \\
\text { Symbol }\end{array}$ & Meaning \\
\hline$C_{p}$ & The cost for periodic checkpointing scheme \\
\hline$\gamma$ & $\begin{array}{l}\text { The normalized cost of checkpointing a } \\
\text { location record according to the paging cost }\end{array}$ \\
\hline$\lambda_{d}$ & MT's Call delivery rate \\
\hline$T_{v}$ & The periodic checkpointing interval \\
\hline$R$ & Call-to-mobility ratio \\
\hline$\lambda_{m}$ & MT's mobility rate \\
\hline$C_{a}$ & $\begin{array}{l}\text { The normalized cost for the aperiodic } \\
\text { checkpointing scheme }\end{array}$ \\
\hline$M$ & Expected number of MTs in the VLR \\
\hline$N_{v}$ & $\begin{array}{l}\text { A threshold value for VLR checkpointing } \\
\text { process }\end{array}$ \\
\hline$C_{a p}$ & $\begin{array}{l}\text { The normalized cost for the proposed aperiodic } \\
\text { checkpointing scheme }\end{array}$ \\
\hline$P_{M}$ & $\begin{array}{l}\text { The probability that a VLR backup information } \\
\text { after the failure is current }\end{array}$ \\
\hline$\delta$ & Penalty factor for call setup delays \\
\hline$N_{R A}$ & Total number of RAs in the VLR \\
\hline$C_{1}$ & $\begin{array}{l}\text { Periodic checkpointing cost when the first event } \\
\text { is a call delivery }\end{array}$ \\
\hline$C_{2}$ & $\begin{array}{l}\text { Periodic checkpointing cost when the first event } \\
\text { is a call origination }\end{array}$ \\
\hline$C_{3}$ & $\begin{array}{l}\text { Periodic checkpointing cost when the first event } \\
\text { is a location registration or confirmation }\end{array}$ \\
\hline$\alpha$ & $\begin{array}{l}\text { Penalty factor for retransmission due to channel } \\
\text { collisions }\end{array}$ \\
\hline$t_{0}$ & Call origination period \\
\hline$t_{d}$ & Interarrival time between call deliveries \\
\hline$t_{p}$ & Timeout period \\
\hline$t_{m}$ & MT's residence time in RAs \\
\hline$T_{p}$ & The periodic location confirmation interval \\
\hline$P_{f}$ & $\begin{array}{l}\text { Expected probability of becoming the location } \\
\text { record obsolete for aperiodic checkpointing } \\
\text { approach }\end{array}$ \\
\hline$C_{f}$ & $\begin{array}{l}\text { Cost to recover the obsolete VLR information } \\
\text { for the aperiodic checkpointing approach }\end{array}$ \\
\hline$C_{f p}$ & $\begin{array}{l}\text { Cost to recover the obsolete VLR information } \\
\text { for the proposed aperiodic checkpointing } \\
\text { approach }\end{array}$ \\
\hline$E\left[N_{R A}\right]$ & $\begin{array}{l}\text { Expected number of RAs in the VLR from } \\
\text { where location information is updated after the } \\
\text { checkpointing process. }\end{array}$ \\
\hline$\pi(i, j)$ & $\begin{array}{l}\text { Stationary probability of state }(i, j) \text { in the steady } \\
\text { state }\end{array}$ \\
\hline$\lambda$ & MT's arrival rate in the VLR \\
\hline$\mu$ & MT's movement rate from the VLR \\
\hline
\end{tabular}




\section{ANALYTICAL MODELING AND EXPERIMENTAL RESULTS}

\subsection{Analytical Modeling}

In this section we model the VLR failure restorations of the periodic checkpointing, aperiodic checkpointing, and the proposed aperiodic checkpointing approaches. We derive the cost of delivering a call to a MT and compare the performance of our proposed aperiodic checkpointing scheme with that of the existing periodic and aperiodic checkpointing schemes. The terms and symbols used for analysis are given in Table 2 .

Suppose that the normalized cost for paging in an RA is 1 . So, the normalized cost for the periodic checkpointing approach under the normal operation is as follows [5].

$$
C_{p}=1+\frac{\gamma}{\lambda_{d} T_{v}}
$$

On the other hand, the normalized cost for the aperiodic checkpointing approach under the normal operation is as follows [5].

$$
\begin{aligned}
C_{a} & =1+\frac{M \gamma}{R N_{v}} \\
& =1+\frac{M \lambda_{m} \gamma}{\lambda_{d} N_{v}}
\end{aligned}
$$

Where, $R=\frac{\lambda_{d}}{\lambda_{m}}[7]$.

Similarly, the normalized cost for the proposed aperiodic checkpointing approach under the normal operation is as follows.

$$
C_{a p}=1+\frac{M \lambda_{m} \gamma}{\lambda_{d} N_{v}}
$$

Alternatively, the VLR restoration after the failure depends on whether the first event is a call delivery or call origination or location confirmation. So, the normalized cost for the periodic checkpointing approach after the failure under this dependency is as follows [5].

$$
\begin{aligned}
C_{p}= & P[X \text { is call delivery }] \times C_{1}+ \\
& P[X \text { is callorigination }] \times C_{2}+ \\
& P[X \text { is location confirmation or registration }] \times C_{3}
\end{aligned}
$$

Where,

$$
\begin{gathered}
C_{1}=P_{M}+\delta\left(1-P_{M}\right) N_{R A}+\frac{\gamma}{\lambda_{d} T_{v}} \\
C_{2}=1+\frac{\gamma}{\lambda_{d} T_{v}} \\
C_{3}=1+\alpha+\frac{\gamma}{\lambda_{d} T_{v}} \\
P_{M}=\frac{1-e^{-\lambda_{m} T_{v}}}{T_{v} \lambda_{m}}
\end{gathered}
$$

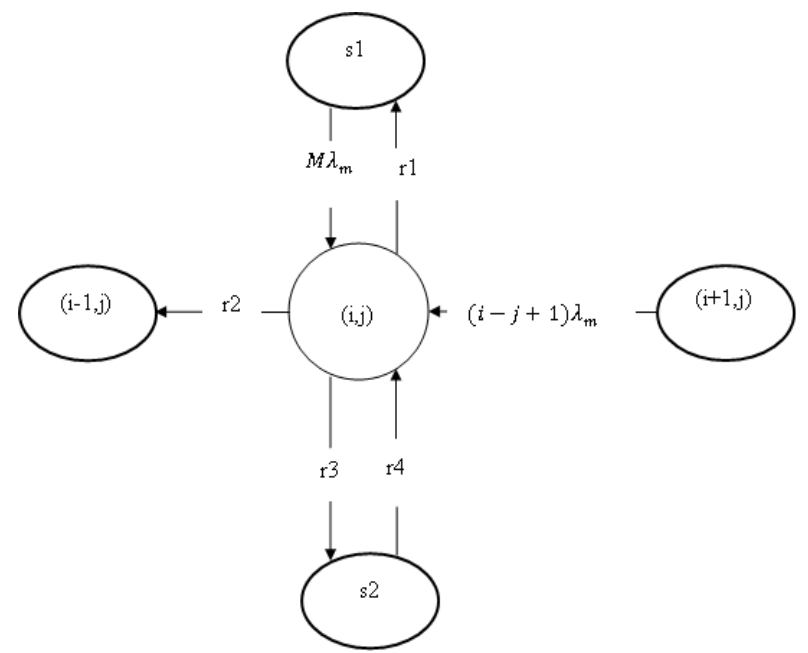

Figure 3. The state diagram for deriving $P_{f}$.

$P[X$ is calldelivery $]$

$=\int_{0}^{\infty} P\left(t_{0}>t_{d}\right) P\left(t_{p}>t_{d}\right) P\left(t_{m}>t_{d}\right) \lambda_{d} e^{-\lambda_{d} t_{d}} d t_{d}$

$=\int_{0}^{\infty} e^{-\lambda_{0} t_{d}} \times \frac{T_{p}-t_{d}}{T_{p}} \times e^{-\lambda_{m} t_{d}} \times \lambda_{d} e^{-\lambda_{d} t_{d}} d t_{d}$

$=\lambda_{d} \int_{0}^{\infty} e^{\left(\lambda_{0}+\lambda_{m}+\lambda_{d}\right) t_{d}} d t_{d}-\frac{\lambda_{d}}{T_{p}} \int_{0}^{\infty} t_{d} e^{-\left(\lambda_{0}+\lambda_{m}+\lambda_{d}\right) t_{d}} d t_{d}$

$=\frac{\lambda_{d}}{\lambda_{0}+\lambda_{m}+\lambda_{d}}-\frac{\lambda_{d}}{T_{p}\left(\lambda_{0}+\lambda_{m}+\lambda_{d}\right)^{2}}$

In the similar way,

$P[X$ is callorigination $]$

$$
=\frac{\lambda_{0}}{\lambda_{0}+\lambda_{m}+\lambda_{d}}-\frac{\lambda_{0}}{T_{p}\left(\lambda_{0}+\lambda_{m}+\lambda_{d}\right)^{2}}
$$

And hence,

$P[X$ is location confirmation or registration $]$

$$
=1-P[X \text { is calldelivery }]-P[X \text { is callorigination }]
$$

On the other hand, the normalized cost for the aperiodic checkpointing approach after the failure under the first event dependency is as follows [5].

$$
C_{a}=\left(1-P_{f}\right)+P_{f} \times C_{f}
$$

Where,

$C_{f}=P[X$ is call delivery $] \times \delta\left(N_{R A}\right)+$

$P[X$ is location confirmation or registration $] \times(1+\alpha)+$

$P[X$ is call origination $]+\frac{M \lambda_{m} \gamma}{\lambda_{d} N_{v}}$ 
which can be derived in the similar way as that of the $C_{p}$ and from the equilibrium equations derived from the state diagram of Figure 3 [5], we get the following equation for $P_{f}$.

$$
P_{f}=\sum_{i, j} \pi(i, j) \times \frac{j}{i}
$$

Similarly, the normalized cost for the proposed aperiodic checkpointing approach after the failure depends on whether the first event is a call delivery or call origination or location confirmation. This cost is the same as that of the equation (12) and can be represented as follows.

$$
C_{a p}=\left(1-P_{f}\right)+P_{f} \times C_{f p}
$$

However, there is a difference in calculating the value of $C_{f}$ and $C_{f p}$. The VLR in the proposed method sends the location confirmation request message to the selected RAs whose location information is obsolete instead of sending it to all the RAs in the VLR. So, the $N_{R A}$ term of equation (13) is replaced by $E\left[N_{R A}\right]$ and can be represented as follows.

$C_{f p}=P[X$ is calldelivery $] \times \delta\left(E\left[N_{R A}\right]\right)+$ $P[X$ is location confirmation or registration $] \times(1+\alpha)+$

$P[X$ is call origination $]+\frac{M \lambda_{m} \gamma}{\lambda_{d} N_{v}}$

Where, it can be derived in the similar way as that of the $C_{p}$ and from the state transition diagram shown in Figure 4 we derive the equation for $E\left[N_{R A}\right]$. Using the concept of local balance, we obtain the following balance equations.

$$
\begin{aligned}
\lambda \pi_{N_{R A}-1} & =\left(\lambda+N_{R A} \mu\right) \pi_{N_{R A}} \\
\lambda \pi_{k} & =(k+1) \mu \pi_{k+1} \quad 1<k<N_{R A} \\
\lambda \pi_{1} & =\lambda \pi_{N_{R A}}+\mu \pi_{2}
\end{aligned}
$$

and

$$
\sum_{i=1}^{N_{R A}} \pi_{i}=1
$$

By solving these equations, we get

$$
\begin{aligned}
& \pi_{N_{R A}}=(2+ \\
& \left.\sum_{k=1}^{N_{R A}-1}\left(\frac{\mu}{\lambda}\right)^{N_{R A}-k-1} \frac{\left(N_{R A}-1\right) !}{k !} \frac{\lambda+\mu N_{R A}}{\lambda}\right)^{-1}
\end{aligned}
$$

$\pi_{k}=\left(\frac{\mu}{\lambda}\right)^{N_{R A}-k-1} \times$

$\frac{\left(N_{R A}-1\right) !}{k !} \frac{\lambda+\mu N_{R A}}{\lambda} \times \pi_{N_{R A}} \quad 1<k<N_{R A}$

$\pi_{1}=\left(1+\left(\frac{\mu}{\lambda}\right)^{N_{R A}-1}\left(N_{R A}-1\right) ! \frac{\lambda+\mu N_{R A}}{\lambda}\right) \times \pi_{N_{R A}}$

Therefore, expected number of RAs in the VLR from where location information is updated after the checkpointing process is as follows.

$$
E\left[N_{R A}\right]=\sum_{i=1}^{N_{R A}} i \times \pi_{i}
$$

\subsection{Experimental Results}

In order to obtain the equivalent experimental results of the proposed and the previous approaches, we used Little's Result ( $N=\lambda \times T$ ) to transform the coordinates of the periodic checkpointing approach to the aperiodic checkpointing approaches. Here, the transformation parameters are $N=N_{v}, \lambda=M \lambda_{m}$, and $T=T_{v}$. Moreover, in order to demonstrate the results, we assume $M=100, N_{R A}=4, \delta=\alpha=2$, and $\gamma=0.5$ and 1.0.

The normalized call delivery cost of the periodic, aperiodic and the proposed aperiodic checkpointing approaches under the normal operation is shown in Figure 5. It shows that the cost of these three approaches for any given CMR under this condition is the same and decreases with the increase of $N_{v}$ or $T_{v}$. This is expected as the equations for these approaches are the same in normal operation after the coordinate transformation. Furthermore, it is observed from Figure 5(a) and 5(b) that these cost decreases with the increase of the CMR. This is also expected and can be explained from the mobility management strategy of the wireless mobile networks. The net call delivery cost decreases if the MT's call delivery rate is higher than the MT's mobility rate as it lowers the called MT's location tracking cost.

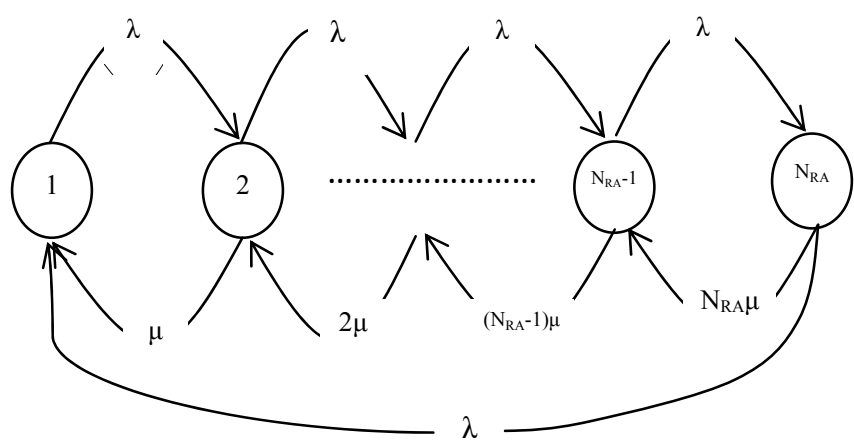

Figure 4. State transition diagram for deriving $E\left[N_{R A}\right]$. 

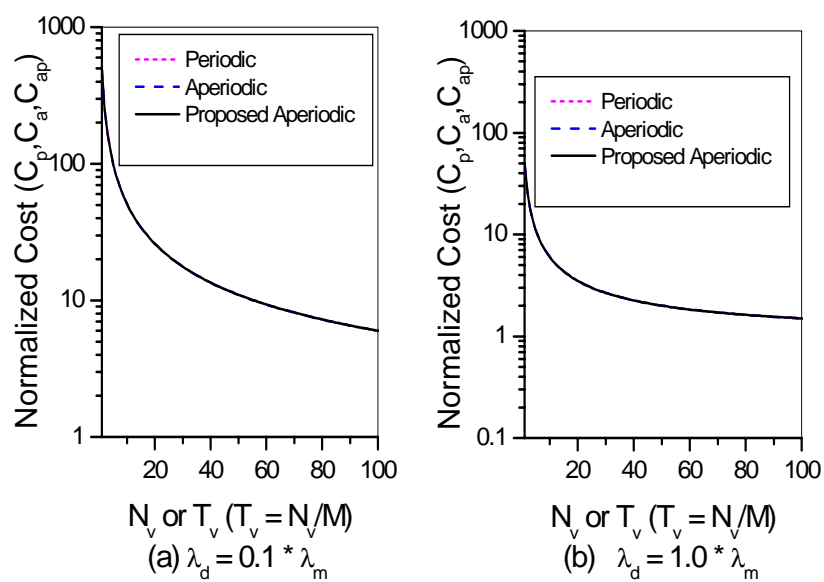

Figure 5. The normalized cost of delivering a call in normal operation.
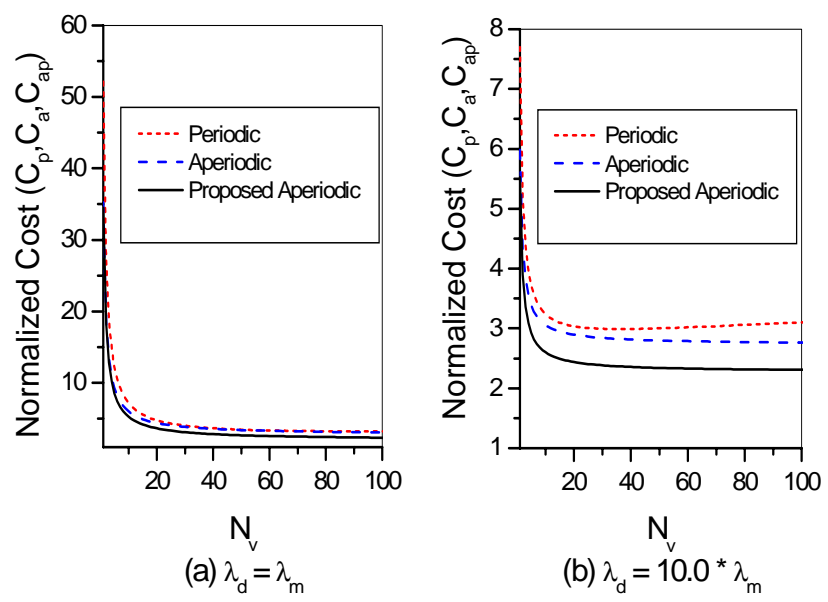

Figure 6. The cost of delivering a call after a VLR failure (when $\gamma=0.5)$.
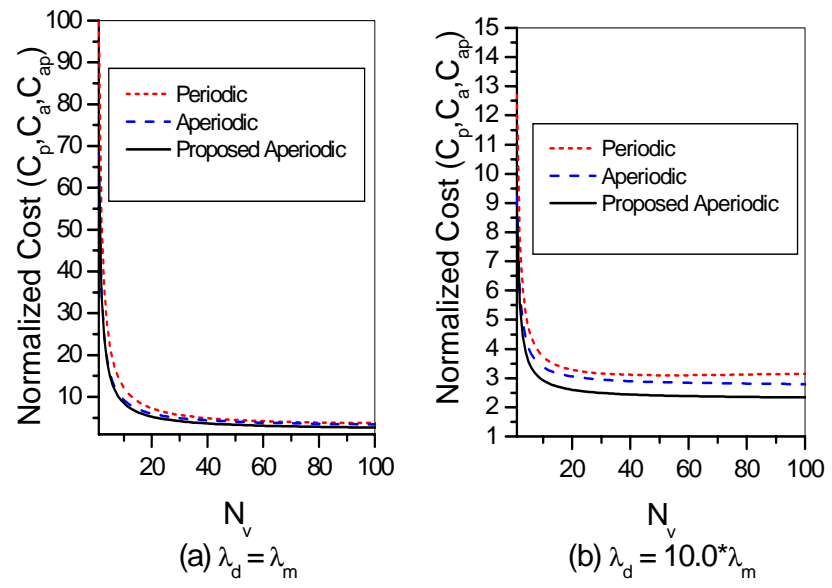

Figure 7. The cost of delivering a call after a VLR failure (when $\gamma=1.0)$.
On the other hand, the normalized call delivery cost of the periodic, aperiodic and the proposed aperiodic checkpointing approaches after the VLR failure is shown in Figure 6 and Figure 7. It is observed from these figures that the proposed method outperforms the other two methods irrespective of the values of $N_{v}, \mathrm{CMR}$, and $\gamma$ and decreases the cost with the increase of $N_{v}$. These trends are expected as the VLR sends the location confirmation request message to the selected RAs for the proposed approach instead of broadcasting this message to all the RAs for the aperiodic checkpointing approach. Whereas for the periodic checkpointing approach; the VLRs are periodically checkpointed irrespective of the number of handoffs in that VLR. As a result, the call delivery cost increases for this approach. Additionally, with the increase of $N_{v}$, the number of VLR checkpointing decreases and therefore the call delivery cost decreases for the three approaches. Moreover, in comparison with the Figure 6(a) and 6(b); 7(a) and 7(b), we observe that the three approaches show lower cost for higher values of the CMR. This is also expected as the total call delivery cost decreases if the MT's call delivery rate is higher than the MT's mobility rate; because it lowers the called MT's location tracking cost. Similarly, if we compare the Figure 6(a) and 7(a); 6(b) and 7(b), we observe that the three approaches show higher cost for higher values of the $\gamma$. The reason behind this tendency is simple the more the cost for checkpointing a location record, the more the call delivery cost.

\section{CONCLUSION}

We have proposed a new selective aperiodic checkpointing approach for VLR failure recovery in wireless mobile networks. In this approach, the VLR databases are backed up in the non-volatile storage when it exceeds the location update counter before its failure. After the failure, it restores the database from the nonvolatile storage, but some of its information may be obsolete due to the location update in that VLR between the time period of back up and failure. In order to solve this problem, a data structure is used to list the RAs whose location information is updated during this time period. The VLR then sends the location confirmation request message selectively to these RAs instead of broadcasting to all RAs. As a result, the total normalized call delivery cost of the proposed method after the failure is reduced than the other approaches. The analytical modeling and numerical results also shows that the proposed method after the failure outperforms the previous methods.

We are currently working on the HLR database failure recovery in order to combine this with the VLR database failure recovery to improve the result of the proposed approach.

\section{REFERENCES}

[1] EIA/TIA. "Cellular Radio-telecommunications Intersystem Operations," Tech. Rep. IS-41 Revision B, EIA/TIA, December 1991. 
[2] M. Mouly and M. B. Pautet, "The GSM System for Mobile Communications," Telecom Publishing, 49 rue Louise Bruneau, Palaiseau, France, January 1992.

[3] Y. B. Lin, "Database Failure Recovery for Cellular Phone Networks," Submitted for publication.

[4] Y. B. Lin, "Failure Restoration of Mobility Databases for Personal Communication Networks," Journal of Wireless Networks, vol. 1, no. 3, pp. 365-372, September 1995.

[5] T. P. Wang, C. C. Tseng, and W. K. Chou, "An Aggressive Approach to Failure Restoration of PCS Mobility Databases," ACM SIGMOBILE Mobile Computing and Communications Review, vol. 1, no. 3, pp. 21-28, September 1997.
[6] S. I. Sou and Y. B. Lin, "Broadcast Approach for UMTS Mobility Database Recovery," IEEE Transactions on Mobile Computing, vol. 6, no. 8, pp. 865-871, August 2007.

[7] R. Jain, Y. B. Lin, C. Lo, and S. A. Mohan, "Caching Strategy to Reduce Network Impacts of PCS," IEEE Journal on Selected Areas in Communications, pp. 1434-1444, October 1994. 